\section{Mass spectrometry in the operating room}

To the Editor:

The department of anaesthesia at the University Hospital in Saskatoon, Saskatchewan recently acquired a mass spectrometer* and thus the ability to do routine anaesthetic gas analysis. Although similar systems have been in use in the United States, ${ }^{\prime}$ this is the first application of a mass spectrometer as a routine operating room monitor in Canada.

A mass spectrometer can separate the components of a gaseous mixture by reproducing a beam of gaseous ions from a sample, and this ionic beam then produces a spectrum according to its mass-tocharge ratio. Analysis of this spectrum allows the calculation of the composition and relative abundance for each ionic species. The initial technical problems of some gases sharing common mass-to-charge ratios (e.g., $\mathrm{CO}_{2}$ and $\mathrm{N}_{2} \mathrm{O}$ ) have been overcome making mass spectrometric measurements very accurate and reliable.

This system can measure the inspiratory and end tidal concentrations of carbon dioxide, nitrogen, nitrous oxide, oxygen and the inhalational agents halothane, enflurane and isoflurane. In our hospital a long nylon line constantly draws gases from the anaesthetic circuit and these gases are brought by vacuum to a central computerized mass spectrometer. The microcomputer interprets and displays the results on remote terminals in each equipped operating room and as hard data at the central station.

The sample gas is drawn at a constant rate creating a lag time of only 0.25 seconds between the source and the mass spectrometer. The response time is the time for steady state to be reached at the mass spectrometer, a requirement for accurate analysis. This time may vary; however, an average of 6-15 seconds has been estimated. Updated information appears at remote terminals in between 15-90 seconds, depending on the numbers of

* SARA (System for Anaesthetic and Respiratory Analysis). Allied Healthcare Products, St. Louis, Missouri. patients monitored. Our equipment has the capacity to monitor 16 sources at one time.

In our experience the mass spectrometer has proved to be an accurate monitor. Our system has its own internal calibration with known concentrations of gases and we have confirmed its accuracy using our own samples.

In our practise it has had many applications. Inspiratory oxygen concentrations from flowmeters and oxygen blenders have been verified. Anacsthetic vapour/gas uptake and washout have been measured. The measurement of nitrogen concentration in the expired air may detect air embolism and we are currently investigating this application.

These systems are designed to be cost competitive with other patient monitors. The initial installation cost of the mass spectrometer, computer and the sampling lines was approximately $\$ 70,000$ (U.S.) Each graphics monitor cost approximately $\$ 2500$ (U.S.) and it is possible to add a printer for hard copy. The maintenance cost estimates vary but a cost comparison between shared mass spectrometers and conventional analyzers has been done. ${ }^{2}$ These systems become cost competitive with isolated monitors when eight or more rooms share one mass spectrometer.

We have found the mass spectrometer to be an accurate and convenient monitor. It has proved a useful teaching aid that enhances the understanding of the dynamics of the anaesthetic gases. The routine use in the operating room of a mass spectrometer makes possible an array of measurements and clinical investigations.

\section{R. Weiler BSC MD}

J.D. Lamb MD FRCP(C)

B.A. Finegan MB FRCs(1)

Department of Anaesthesia

University Hospital

Saskatoon, Saskatchewan

\section{REFERENCES}

1 Ozanne GM, Young WG, Mazzel WJ. Multipatient anesthetic mass spectrometry. Anesthesiology 1981; 55: 62-70.

2 Swedlow $D B$. The role of mass spectrometry in the operating room, post-anesthesia recovery and intensive care unit. 1984 Annual Refresher Course Lectures, American Society of Anesthesiologists. 